\title{
Improved bounds on the max-flow min-cut ratio for multicommodity flows *
}

\author{
Serge Plotkin ${ }^{\dagger}$ \\ Stanford University
}

\author{
Éva Tardos ${ }^{\ddagger}$ \\ Cornell University
}

\begin{abstract}
In this paper we consider the worst case ratio between the capacity of min-cuts and the value of max-flow for multicommodity flow problems. We improve the best known bounds for the mincut max-flow ratio for multicommodity flows in undirected graphs, by replacing the $O(\log D)$ in the bound by $O(\log k)$, where $D$ denotes the sum of all demands, and $k$ denotes the number of commodities. In essence we prove that up to constant factors the worst min-cut max-flow ratios appear in problems where demands are integral and polynomial in the number of commodities.

Klein, Rao, Agrawal, and Ravi have previously proved that if the demands and the capacities are integral, then the min-cut max-flow ratio in general undirected graphs is bounded by $O(\log C \log D)$, where $C$ denotes the sum of all the capacities. Tragoudas has improved this bound to $O(\log n \log D)$, where $n$ is the number of nodes in the network. Garg, Vazirani and Yannakakis further improved this to $O(\log k \log D)$. Klein, Plotkin and Rao have proved that for planar networks, the ratio is $O(\log D)$.

Our result improves the bound for general networks to $O\left(\log ^{2} k\right)$ and the bound for planar networks to $O(\log k)$. In both cases our result implies the first non-trivial bound that is independent of the magnitude of the numbers involved. The method presented in this paper can be used to give polynomial time approximation algorithms to the minimum cuts in the network up to the above factors.
\end{abstract}

\footnotetext{
*Preliminary version appeared in Proc. 25th Symp. on Theory of Computing, 1993.

${ }^{\dagger}$ Research supported by U.S. Army Research Office Grant DAAL-03-91-G-0102, and by a grant from Mitsubishi Electric Laboratories.

${ }^{\ddagger}$ Research supported in part by a Packard Fellowship, an NSF PYI award, a Sloan Fellowship, and by the National Science Foundation, the Air Force Office of Scientific Research, and the Office of Naval Research, through NSF grant DMS-8920550.
} 


\section{Introduction}

The multicommodity flow problem involves simultaneously shipping several different commodities from their respective sources to their sinks in a single network so that the total amount of flow going through each edge does not exceed its capacity. Each commodity has an associated demand, which is the amount of the commodity that we wish to ship.

Given a multicommodity flow problem, one often wants to know if there is a feasible flow, i.e. a flow which both satisfies the demands and obeys the capacity constraints. In order to prove that such flow does not exist, it is sufficient to exhibit a cut whose capacity is below the sum of the demands that are separated by the cut. The min-cut max-flow theorem for the single-commodity flow problem [3] states that the non-existence of such a "bad" cut proves that a feasible flow does exist. In contrast to this, a multicommodity flow problem can be infeasible even if the cut condition is satisfied. The simplest such example is where the network is $K_{2,3}$ with unit-capacity edges and unit demand between every pair of nodes not connected by an edge.

A natural question to ask is how large a safety margin do we need, i.e. how large should be the minimum ratio (over all cuts) of the capacity of the cut to the sum of the demands that are separated by this cut, in order to ensure existence of a feasible flow. In the next section we will formally define the max-flow and min-cut problems for multicommodity flows, and we will see that the minimum required safety margin is exactly the maximum (over all multicommodity flow problems) of the min-cut max-flow ratio.

A related problem is to consider a multicommodity flow problem, and to search either for a feasible flow, or for a cut whose ratio is below the above mentioned safety margin. This leads to an algorithm that finds an approximately minimum cut. Approximately minimum cut computation is a basic step for construction of approximation algorithms for a variety of NP-complete problems [9, 7]. However, in these applications, the required min-cut is for the unit-demand case.

Previous studies of min-cut max-flow relationships in multicommodity flow problems concentrated on finding special classes of problems for which exact min-cut max-flow theorem holds (see for example, $[11,10])$. Leighton and Rao were the first to prove bounds on the min-cut max-flow ratio for multicommodity flow problems in general graphs. In their pioneering work [9] they considered multicommodity flow problems in general graphs, where there is one unit of demand between every pair of nodes. We will refer to this case as a uniform-demand multicommodity flow problem. They proved that the min-cut max-flow ratio in this case is at most $O(\log n)$. Alternatively, this means that there exists a safety margin factor bounded by $O(\log n)$, such that in order to prove existence of a feasible solution in the uniform-demand case, it is sufficient to show that the capacity of every cut exceeds the demand across the cut by at least this factor. This result holds both for directed and undirected networks. Leighton and Rao have also shown that $O(\log n)$ ratio is the best possible. They also provide a polynomial time $O(\log n)$-approximation algorithm to the min-cut problem. The algorithm first solves the corresponding uniform-demand multicommodity flow problem (and its linear programming dual) and uses the solution to find an approximately minimum cut.

Klein, Rao, Agrawal, and Ravi [7] were the first to prove a bound on the min-cut max-flow ratio in general undirected multicommodity flow networks. They proved that this ratio is bounded 
by $O(\log C \log D)$, where $C$ and $D$ denote the sum of all the capacities and the sum of all the demands, respectively, assuming that both capacities and demands are integral. Tragoudas $[14,13]$ has improved the bound to $O(\log n \log D)$, and Garg, Vazirani and Yannakakis[4] have further improved it to $O(\log k \log D)$. Klein, Plotkin and Rao [5] have shown that the ratio in planar graphs is bounded by $O(\log D)$, and in the planar uniform-demand case by $O(1)$. Observe that using the (single commodity) max-flow min-cut theorem $k$ times we get that the ratio is at most $k$, and therefore the above bounds are weak for the case where the demands are very large.

We show that in order to find an approximate minimum cut, it is sufficient to look for such cuts in several transformed problems, each of which has integral demands whose sum $D$ is bounded by a small-degree polynomial in $k$, the number of commodities in the original problem. Using the results of $[9,7,14,4,5]$ mentioned above, we show that the min-cut max-flow ratio for general multicommodity flow problems is bounded by $O\left(\log ^{2} k\right)$, and for the planar case by $O(\log k)$. Moreover, our results can be used to find approximately minimum cuts. We say that a minimum cut algorithm is a $\rho$-approximation algorithm if it is guaranteed to find a cut whose value is at most $\rho$ times the optimal value. We show that there exists a polynomial time $O\left(\log ^{2} k\right)$-approximation algorithm for finding the min-cut in general networks and a polynomial time $O(\log k)$-approximation algorithm in planar networks. These are the first such bounds that are independent of the size of the demands and do not require any integrality assumptions.

It is interesting to note that much less is known about the min-cut max-flow ratio for directed multicommodity flow problems. Leighton and Rao proved a polylogarithmic bound for the special case of uniform demands, implying a polylogarithmic approximation algorithm for the feedback arcset problem, Seymour [12] improved the later bound. Polylogarithmic bounds for min-cut max-flow ratios for symmetric directed multicommodity flow (where the flow from source to sink has to be equal to the flow from sink back to the source) with arbitrary demands were shown in [6] and, later, in [2]. It is an interesting open problem to extend the results for general undirected multicommodity flows to the general (non-symmetric) directed case.

\section{Definitions and Notation}

An instance of the multicommodity flow problem consists of an undirected graph $G=(V, E)$, a nonnegative capacity $u(v w)$ for every edge $v w \in E$, and a specification of $k$ commodities, numbered 1 through $k$, where the specification for commodity $i$ consists of a source-sink pair $s_{i}, t_{i} \in V$ and a non-negative demand $d_{i}$. We will denote the number of nodes by $n$, and the number of edges by $m$.

A flow $f_{i}$ of commodity $i$ in $G$ from node $s_{i}$ to node $t_{i}$ is defined as a collection of paths from $s_{i}$ to $t_{i}$, with associated real values. Let $\mathcal{P}_{i}$ denote a collection of paths from $s_{i}$ to $t_{i}$ in $G$, and let $f_{i}(P)$ be a nonnegative value for every $P$ in $\mathcal{P}_{i}$. The value of the flow thus defined is $\sum_{P \in \mathcal{P}_{i}} f_{i}(P)$, which is the total flow delivered from $s_{i}$ to $t_{i}$. The amount of flow of commodity $i$ through an edge $v w$ is given by

$$
f_{i}(v w)=\sum\left\{f_{i}(P): P \in \mathcal{P}_{i} \text { and } v w \in P\right\}
$$


A feasible multicommodity flow $f$ in $G$ consists of a flow $f_{i}$ from $s_{i}$ to $t_{i}$ of value $d_{i}$ for each commodity $1 \leq i \leq k$. We require that $f(v w) \leq u(v w)$ for every edge $v w \in E$, where we use $f(v w)=\sum_{i=1}^{k} f_{i}(v w)$ to denote the total amount of flow on edge $v w$.

A natural maximization version of the problem is to determine the maximum percentage $z^{*}$ such that at least $z^{*}$ percent of each demand can be shipped without violating the capacity constraints. We will refer to this problem, also known as the concurrent flow problem, as the max-flow problem for multicommodity flows. It is equivalent to the problem of determining the minimum ratio $\lambda^{*}$ such that $100 \%$ of each one of the demands can be shipped in the network with capacities $\lambda^{*} u(e)$. We will refer to $\lambda^{*}$ as the congestion of the network. Clearly, $\lambda^{*} z^{*}=1$.

Given a subset $U$ of the nodes, let the capacity of the corresponding cut be defined by:

$$
u(U)=\sum_{v \in U, w \in \bar{U}} u(v w)
$$

Similarly, define the demand across the cut $(U, \bar{U})$ :

$$
d(U)=\sum_{i:\left\{s_{i}, t_{i}\right\} \cap U \mid=1} d_{i}
$$

If there exists a feasible flow satisfying demands $z d_{i}$, then we must have that $z$ times the demand across any cut is at most as much as the capacity of the cut, $z d(U) \geq u(U)$. This motivates the definition of the min-ratio cut, or min-cut, for short:

$$
S^{*}=\min _{U \subset V} \frac{u(U)}{d(U)}
$$

By the above, the min-cut value $S^{*}$ is at least as big as the max-flow value $z^{*}$. Clearly, the maximum (over a class of multicommodity flow problems) of the min-cut max-flow ratio is exactly the minimum required safety margin necessary to imply the existence of a feasible flow. Formally, the result in [9] means that for the uniform-demand case, $S^{*} / z^{*}=O(\log n)$; combination of the results in [7], [14], and [4] mean that, in general, $S^{*} / z^{*}=O(\log k \log D)$; the results in [5] mean that in planar graphs, $S^{*} / z^{*}=O(\log D)$, and in the planar uniform-demand case $S^{*} / z^{*}=O(1)$, where $D$ denotes the sum of all demands assuming that demands are integral. In the next section we show that $S^{*} / z^{*}=O\left(\log ^{2} k\right)$ in general networks and $O(\log k)$ in planar ones without assuming integrality of the demands.

\section{Min-Ratio Cuts in Networks with Large Demands}

In this section we prove the main result of this paper, that up to a small constant factor the worst min-cut max-flow ratio is obtained on graphs with integer demands where $D$, the sum of all demands, is bounded by a small-degree polynomial in $k$. Since we are looking only for approximately minimum cuts, it is natural to round both demands and capacities. However, note that with traditional 
rounding techniques it seems hard to handle the case when the ranges of the different demands and the different capacities are both large. One cannot a priori know whether a cut with large demand and large capacity or one with small demand and small capacity is more congested. In particular, we cannot disregard even the smallest capacity edges.

Our main proof technique is as follows. We separate the demands into groups, each consisting of commodities of roughly comparable demand. The traditional rounding technique shows that the min-cut max-flow ratio in a problem induced by the demands in a single group depends only on $\log k$ instead of $\log D$. The main result is that both the min-cut and the max-flow in the whole network are within a small constant factor of the corresponding values in one of the groups. This will imply that the min-cut and the max-flow are within the claimed $O\left(\log ^{2} k\right)$ factor (or $O(\log k)$ factor in planar graphs) in the whole network. Moreover, this also implies that one can find an approximation to within the above factors to the min-cut in the whole network by finding such approximately minimal cuts in each of the groups.

Let $d_{\min }$ and $d_{\max }$ denote the minimum and maximum non-zero demand. A simple rounding technique shows that $\log D$ in the results about the min-cut max-flow ratio listed in the introduction can be replaced by $\log \hat{D}$ where $\hat{D}=\sum_{i} d_{i} / d_{\text {min }}$; this new bound holds without assuming that the demands are integral.

Lemma 3.1 In a multicommodity flow problem in general graphs $S^{*} / z^{*}=O(\log \hat{D} \log k)$. In planar graphs $S^{*} / z^{*}=O(\log \hat{D})$. There exists a polynomial time $O(\log k \log \hat{D})$-approximation algorithm for finding the min-cut in general networks and an $O(\log \hat{D})$-approximation algorithm in planar networks.

Proof: Round all demands up to integer multiples of $d_{\min }$. Rounding up cannot increase $z^{*}$. The demand of a commodity increases by at most a factor of 2 , and therefore $S^{*}$ decreases by at most a factor of 2. Let $S^{\prime}$ and $z^{\prime}$ denote the min-cut and the max-flow values in the rounded problem. The rounded problem, when demands are scaled by a factor of $d_{\text {min }}$, has integer demands that sum to at most $\hat{D}+k \leq 2 \hat{D}$. Using the theorem of Klein, Rao, Agrawal, and Ravi [7], we have the following chain of inequalities:

$$
\begin{aligned}
S^{*} & \leq 2 S^{\prime} \\
& \leq O\left(z^{\prime} \log k \log (2 \hat{D})\right) \\
& \leq O\left(z^{*} \log k \log \hat{D}\right) .
\end{aligned}
$$

Similarly, using the theorem of Klein, Plotkin and Rao [5] we get that $S^{*} / z^{*}=O(\log \hat{D})$ in planar graphs. This proves the claimed bounds on the min-cut max-flow ratio. To get the polynomial time approximation algorithms we use the approximation algorithms in $[7,5]$ on the rounded network, and note that the capacity/demand ratio of a cut is not changed by more than a factor of 2 by rounding.

Corollary 3.2 If $d_{\max } / d_{\min }=O(k)$ in a multicommodity flow problem, then $S^{*} / z^{*}=O\left(\log ^{2} k\right)$ for general networks, and $S^{*} / z^{*}=O(\log k)$ for planar networks. 
Now consider a multicommodity flow problem with demands that are of greatly differing sizes. Divide the commodities into groups, such that the first group $Q_{1}$ consists of commodities that have demand between $d_{\min }$ and $4 k d_{\min }$; the $i$ th group $Q_{i}$ consists of commodities with demands between $(4 k)^{i-1} d_{\min }$ and $(4 k)^{i} d_{\min }$. Let $z_{i}^{*}, \lambda_{i}^{*}$, and $S_{i}^{*}$ denote the max-flow value, the minimum congestion, and the min-cut, respectively, in the multicommodity flow problem where we consider only commodities in $Q_{i}$. Recall that $\lambda_{i}^{*}=1 / z_{i}^{*}$. Clearly, $\max _{i} \lambda_{i}^{*} \leq \lambda^{*}$, and $\min _{i} S_{i}^{*} \geq S^{*}$. The following two theorems will imply our main result.

Theorem 3.3 In the multicommodity flow problem with the decomposition defined above, $\min _{i} S_{i}^{*} \leq$ $2.25 S^{*}$.

Proof: Let the set $U \subseteq V$ define the cut that achieves ratio $S^{*}$, i.e. the most congested cut in the whole network. Let $d_{j}(U)$ denote the demand in group $j$ across the cut defined by $U$, and let $i$ be the maximum index such that $d_{i}(U)$ is non-zero. The total demand across cut $U$ can be written as:

$$
\begin{aligned}
d(U) & =d_{i}(U)+d_{i-1}(U)+\sum_{j<i-1} d_{j}(U) \\
& \leq \frac{5}{4} d_{i}(U)+d_{i-1}(U),
\end{aligned}
$$

where the inequality follows from the fact that $d_{i}(U)$ is non-zero, and that the demand of any commodity in group $i$ is at least 4 times the sum of all the demands in groups $j<i-1$. This inequality on $d(U)$ implies the following chain of inequalities on $S^{*}$.

$$
\begin{aligned}
S^{*} & =\frac{u(U)}{d(U)} \\
& \geq \frac{u(U)}{(5 / 4) d_{i}(U)+d_{i-1}(U)} \\
& \geq \frac{4}{9} \min \left(\frac{u(U)}{d_{i}(U)}, \frac{u(U)}{d_{i-1}(U)}\right) \\
& \geq \frac{4}{9} \min \left(S_{i}^{*}, S_{i-1}^{*}\right) .
\end{aligned}
$$

This proves the theorem.

Observe that Theorem 3.3, Corollary 3.2, and the known approximation algorithms for the minimum cut problem are enough to give polynomial time approximation algorithms for the minimum cut problem with the improved performance guarantee.

Corollary 3.4 There exists a polynomial time $O\left(\log ^{2} k\right)$-approximation algorithm for the minimum cut in undirected networks, and an $O(\log k)$-approximation algorithm for planar networks.

Remark: Notice that in order to find a cut that is within the above $O\left(\log ^{2} k\right)$ (or $O(\log k)$ for planar graphs) factor to the min-cut, we have to find such approximately minimum cuts in the problems defined by each of the commodity groups. The most time consuming part of the approximation 
algorithms $[7,4,5]$ in both cases is approximately solving the corresponding multicommodity flow problem (and its linear programming dual). The currently known fastest approximation algorithms for the multicommodity flow problem ([8] for general graphs and [1] for the case when the flow paths are very short), run in time proportional to the number of commodities. Therefore, the time required to find approximate optimal flows for each of the subproblems defined by the different groups of demands $Q_{i}$ for $i=1,2, \ldots$, is the same as approximately solving the original multicommodity flow problem alone.

Theorem 3.5 In a multicommodity flow problem with the decomposition defined above, $\lambda^{*} \leq 4 \max _{i} \lambda_{i}^{*}$.

Before we turn to the proof of this theorem we consider its implications. Theorem 3.5 implies the main claim of this paper.

Corollary 3.6 In a general multicommodity flow problem $S^{*} / z^{*}=O\left(\log ^{2} k\right)$, and in planar networks $S^{*} / z^{*}=O(\log k)$.

Proof: Theorem 3.5 implies that $z^{*} \geq(1 / 4) \min z_{i}^{*}$. Together with the trivial inequality $S^{*} \leq$ $\min _{i} S_{i}^{*}$ this gives the following chain of inequalities:

$$
\begin{aligned}
S^{*} & \leq \min _{i} S_{i}^{*} \\
& \leq O\left(\log ^{2} k\right) \cdot \min _{i} z_{i}^{*} \\
& \leq O\left(\log ^{2} k\right) \cdot z^{*} .
\end{aligned}
$$

In planar graphs the $O\left(\log ^{2} k\right)$ factor can be replaced by $O(\log k)$.

To prove Theorem 3.5 we first consider the following lemma.

Lemma 3.7 Consider two multicommodity flow problems defined over the same graph with the same capacities, where the first problem has to ship commodities in group $Q$ and the second problem has to ship commodities in group $Q^{\prime}$. Assume there exist feasible multicommodity flows for the first and the second problems. If every commodity in group $Q^{\prime}$ has demand at least 4 times the total demand of all the commodities in $Q$, then there exists a feasible flow that simultaneously satisfies the demands of commodities in $Q$ and at least half of each of the demands of the commodities in $Q^{\prime}$.

Proof: Let $f$ and $f^{\prime}$ denote the feasible flows of the problems associated with the commodities in groups $Q$ and $Q^{\prime}$, respectively. It is no loss of generality to assume that both of the flows $f$ and $f^{\prime}$, and the capacities $u$ are rational. Multiplying up with the common denominator, we can further assume without loss of generality that $f, f^{\prime}$, and $u$ are integral. We will regard an edge $e$ with 


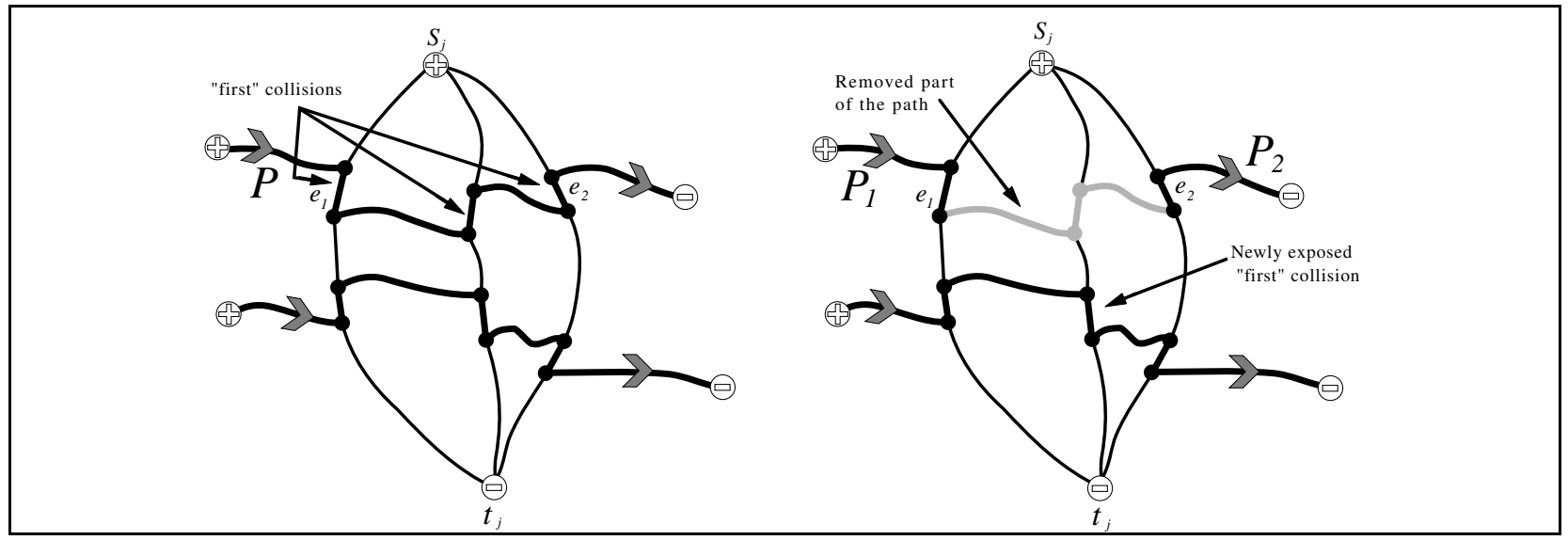

Figure 1: Reducing the number of collisions.
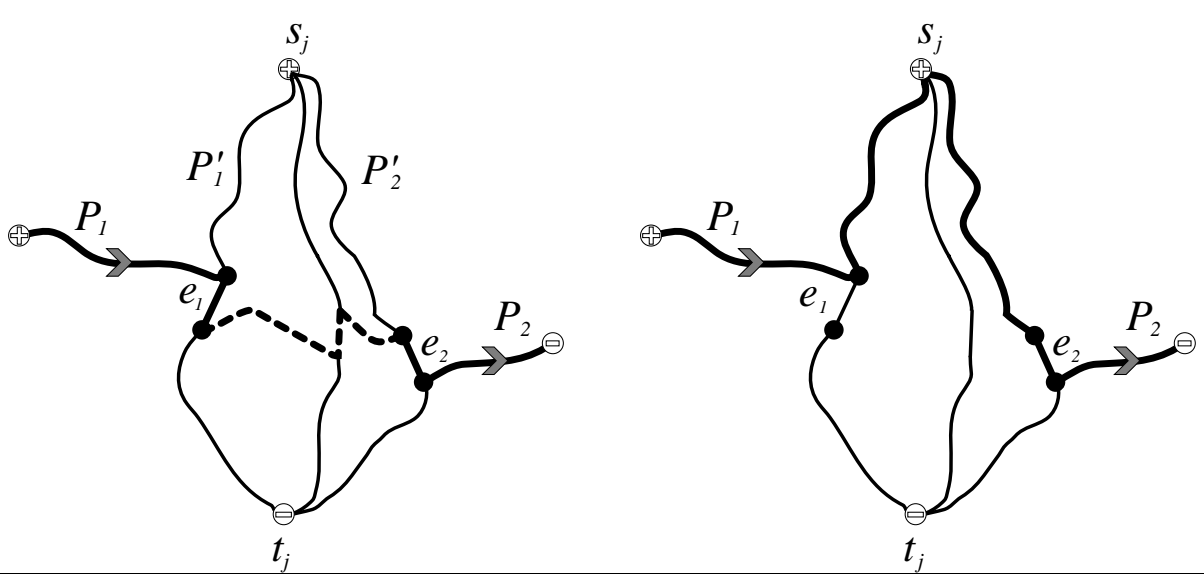

Figure 2: Rerouting flow of one set of commodities using the paths of the other set.

capacity $u(e)$ as a collection of $u(e)$ parallel edges, and the flows $f$ and $f^{\prime}$ as collections of edgedisjoint simple paths in the network. Notice that nothing prevents a flow path of a commodity in $Q$ from using the same edge as some flow path of a commodity in $Q^{\prime}$. We will call such situation a collision. The idea of the proof is to delete a small number of flow paths of commodities in $Q^{\prime}$ and reroute the flow paths of the commodities in $Q$ in order to eliminate all collisions. We will eliminate collisions one at a time, giving a pseudopolynomial algorithm for rerouting. (Note, however, that the flow, whose existence is proved by this lemma, can be constructed without referring to this proof, by running a multicommodity flow algorithm.)

We will first show how to eliminate collisions with a single commodity $j \in Q^{\prime}$. On each flow path of commodity $j$, we will note the first collision and denote the set of these "first collision edges" by $F_{j}$. Consider a flow path $P$ of some commodity in $Q$ that uses more than 2 edges of $F_{j}$, i.e. $\left|P \cap F_{j}\right|>2$ (see Figure 1). Let $e_{1}$ be the first edge in $P$ that is also in $F_{j}$ and let $e_{2}$ be the last such edge, where the order is according to the appearance of these edges in $P$. Delete all edges between $e_{1}$ and $e_{2}$ from $P$. Although instead of a single path $P$ we now have two half-paths $P_{1}$ and $P_{2}$, we 
will still consider them as a single entity and will retain the ordering of the edges as in the original $P$.

Deleting edges from some flow path $P$ of a commodity in $Q$ changes $F_{j}$, by exposing new collisions as the first ones on some flow paths of commodity $j$ (see Figure 1). However, the number of collisions is decreasing, and hence after a finite number of such changes the set $F_{j}$ will contain at most two collision edges on each of the flow paths of the commodities in $Q$. Now delete all the flow paths of commodity $j$ that cause collisions. Clearly, this eliminates all the collisions caused by commodity $j$. Every flow path of a commodity in $Q$ is first on at most 2 flow paths of commodity $j$, and therefore the total flow carried by the flow paths of commodity $j$ that were removed is at most twice the total flow of all the commodities in $Q$. The assumptions in the claim of the lemma imply that this amount does not exceed $d_{j} / 2$, and hence the remaining flow paths of $j$ carry at least $d_{j} / 2$ flow, i.e. satisfying at least half of the demand.

Some of the flow paths of commodities in $Q$ were broken into two halves by the above procedure. It remains to show how to reconnect these paths without causing any new collisions. Consider a flow path $P$ of a commodity in $Q$. The remaining part of $P$ consists of two half paths $P_{1}$ and $P_{2}$ (see Figure 2). By construction, the last edge of $P_{1}$ (marked as $e_{1}$ on the figure) was the first collision edge on a flow path $P_{1}^{\prime}$ of commodity $j$. Similarly, the first edge in $P_{2}$ (marked $e_{2}$ ) is the first collision edge on another flow path $P_{2}^{\prime}$ of commodity $j$. (Dashed line marks the part of $P$ that was deleted as we have described above.) We will use the part of the path $P_{i}^{\prime}$ for $i=1,2$ between the source of commodity $j$ and $e_{i}$ to connect the loose ends of $P_{1}$ and $P_{2}$. This will not create new collisions since $e_{1}$ and $e_{2}$ were the first collisions on the paths $P_{1}^{\prime}$ and $P_{2}^{\prime}$, and the paths of the commodities in $Q$ are edge-disjoint.

Observe that if $Q^{\prime}$ contains more than one commodity, the above process of flow rerouting can be repeated for each commodity.

Proof of Theorem 3.5: Let $\lambda=\max _{i} \lambda_{i}^{*}$. First we consider all of the commodities in $Q_{i}$ with even $i$. For every such group $Q_{i}$, we have a multicommodity flow $f_{i}$ satisfying the capacity constraints $\lambda u(e)$ on every edge $e$. We claim that there exists a multicommodity flow $f_{\text {even }}$ that satisfies the capacity constraints $\lambda u(e)$ on every edge $e$, and satisfies at least half of the demand of every commodity in groups $Q_{i}$ for even $i$.

We prove the above claim by induction on the number of even-indexed groups. The claim is obviously true for $i=2$. To prove that the required flow exists for some $i>2$, apply Lemma 3.7 for commodity groups $Q=Q_{2} \cup \ldots \cup Q_{i-2}$, and $Q^{\prime}=Q_{i}$. Inductively, assume that there exists a flow $f$ that satisfies at least half of the demand of each commodity in $Q$. Lemma 3.7 applied to $f$ and $f_{i}$ implies that there exists a flow that satisfies all the demands that were satisfied by $f$ and at least half of each one of the demands of the commodities in $Q^{\prime}$.

Applying the same argument for the sets $Q_{i}$ for odd $i$, we conclude that there exist flows $f_{\text {even }}$ and $f_{\text {odd }}$ such that together they satisfy at least half of each demand. Moreover, both $f_{\text {even }}$ and $f_{\text {odd }}$, separately, satisfy capacity constraints $\lambda u(e)$. Therefore, there exists a flow $f$ that satisfies all of the demands and satisfies capacity constraints $4 \lambda u(e)$. 


\section{References}

[1] B. Awerbuch and T. Leighton. Improved approximation algorithms for the multi-commodity flow problem and local competitive routing in dynamic networks. In Proc. 26th Annual ACM Symposium on Theory of Computing, pages 487-495, 1994.

[2] G. Even, J. Naor, B. Schieber, and M. Sudan. Approximating minimum feedback sets and multi-cuts in directed graphs. Unpublished manuscript, May 1994.

[3] L. R. Ford, Jr. and D. R. Fulkerson. Flows in Networks. Princeton Univ. Press, Princeton, NJ, 1962.

[4] N. Garg, V. V. Vazirani, and M. Yannakakis. Approximate max-flow min-(multi)cut theorems and their applications. In Proc. 25th Annual ACM Symposium on Theory of Computing, May 1993.

[5] P. Klein, S. Plotkin, and S. Rao. Planar graphs, multicommodity flow, and network decomposition. In Proc. 25th Annual ACM Symposium on Theory of Computing, May 1993.

[6] P. Klein, S. Plotkin, S. Rao, and E. Tardos. Bounds on the max-flow min-cut ratio for directed multicommodity flows. Technical Report CS-93-30, Brown University, September 1993.

[7] P. N. Klein, S. Rao, A. Agrawal, and R. Ravi. An approximate max-flow min-cut relation for multicommodity flow, with applications. Combinatorica, to appear. Preliminary version appeared as "Approximation through multicommodity flow," In Proc. 31th IEEE Annual Symposium on Foundations of Computer Science, pages 726-737, 1990.

[8] T. Leighton, F. Makedon, S. Plotkin, C. Stein, É. Tardos, and S. Tragoudas. Fast approximation algorithms for multicommodity flow problem. In Proc. 23th ACM Symposium on the Theory of Computing, pages 101-111, May 1991.

[9] T. Leighton and S. Rao. An approximate max-flow min-cut theorem for uniform multicommodity flow problems with applications to approximation algorithms. In Proc. 29th IEEE Annual Symposium on Foundations of Computer Science, pages 422-431, 1988.

[10] H. Okamura and P.D. Seymour. Multicommodity flows in planar graphs. J. Combinatorial Theory (B), 31:75-81, 1981.

[11] P.D. Seymour. Matroids and multicommodity flows. European Journal of Combinatorics, 2:257-290, 1981 .

[12] P.D. Seymour. Packing directed circuits fractionally. Unpublished manuscript. Revised November 1993, June 1992.

[13] S. Tragoudas. Improved approximations for the min-cut ratio and the flux. Mathematical Systems Theory, to appear.

[14] S. Tragoudas. VLSI partitioning approximation algorithms based on multicommodity flow and other techniques. PhD thesis, University of Texas at Dallas, 1991. 